\title{
Postural determinants of frontal-position advantage in listening to speech
}

\author{
JOSÉ MORAIS, LUZ CARY, HÉLÈNE VANHAELEN, and PAUL BERTELSON \\ Laboratoire de Psychologie expérimentale, Université libre de Bruxelles, 1050 Brussels, Belgium
}

\begin{abstract}
Previous work has shown that when a subject is seated with body, head, and eyes oriented in the same direction, speech coming from the front is better perceived than speech coming from other directions. The question asked was which segments of the body are critical in determining the advantage of the frontal position. It was found that the effect does not depend exclusively on the orientation of the head relative to the source, since the advantage is reduced not only when the head is deviated laterally towards a competing source, but also when the gaze or the trunk and the limbs are deviated in that direction. Frontal position advantage is thus not a purely acoustical or auditory phenomenon, but depends, at least partly, on interactions at deeper levels.
\end{abstract}

Considerable attention has been devoted in recent years to lateral asymmetries in auditory perception. In dichotic situations, speech delivered to the right ear is identified more accurately than speech delivered simultaneously to the left ear (Kimura, 1961; StuddertKennedy, \& Shankweiler, 1970), while the opposite asymmetry has been reported in the recognition of other classes of sound (Curry, 1967; Kimura, 1964). Interest in these phenomena has been stimulated mainly by their demonstrated relation to hemispheric specialization of function (Kimura, 1961). On the other hand, several essential questions regarding the mechanism and the origin of auditory lateral asymmetries are still completely unanswered.

One reason for this state of affairs might be the quasi-exclusive concentration of experimental work on the dichotic situation. From a biological point of view, dichotic listening is a completely artificial situation which appeared in man's history only with the introduction of the two-channel tape recorder in psychological laboratories. If lateral asymmetry played some role in the development of hemispheric specialization, it must have been through its manifestation in ecologically more valid situations such as those occurring in free-field situations where all sounds reach the two ears, but sometimes with intensity and time-of-arrival interaural differences.

Another unfortunate consequence of concentration on dichotic listening is that interpretations applicable only to that situation have been developed. The dominant interpretation of right-ear advantage, first formulated by Kimura $(1961,1967)$ and subsequently elaborated by Sparks and Geschwind (1968), is one based on the anatomy of the ascending path-

This work was partially supported by the Belgian "Fonds de la Recherche Fondamentale Collective" (Contract 10.152). Luz Cary is now at Universidade de Lisboa. ways connecting the ears to the temporal lobes of the two hemispheres. It offers no room for auditory localization which is such a prominent function of the binaural system.

For those reasons, a program of research has been started in this laboratory to study spatial position effects in free-field situations. Morais and Bertelson (1973) found that when speech was delivered simultaneously on two loudspeakers, one to the left and one to the right of a listener, the messages coming on the right were better identified. When these authors combined a loudspeaker in front of the listener with another to his left or to his right, the frontal message was always recalled better. Later, Hublet, Morais, and Bertelson (1976) attempted a more thorough examination of spatial constraints on attention focusing. They presented pairs of simultaneous speech messages, one from a loudspeaker in a frontal position and another from a loudspeaker in one of several other azimuthal locations around the listener. They found better performance for the frontal message, the superiority being stronger when the competing message came from the left than when it came from the right and increasing with increasing angular separation of the second loudspeaker from the frontal one. In another study, the same authors (Hublet, Morais, \& Bertelson, 1977) found the same pattern of performance, that is, better identification of frontal messages than of posterior ones and of messages from the right than of messages from the left, in a situation where two messages, one to be recalled and one to be ignored, were delivered simultaneously in the same spatial position.

By themselves, these findings are not incompatible with an interpretation in terms of acoustical (intensity of the messages at the ears) and auditory (anatomy of the ear-hemisphere pathways) factors. Right-side advantage might be due to the fact that the right ear was stimulated by the sound coming 
from the right at a higher intensity level than by the message coming from the left. And frontal-position advantage might be due to the fact that a sound coming from the front reaches both ears without obstacle,' while a sound coming from one side meets the head before reaching the ear on the other side and a sound coming from the rear meets the pinna before entering the meatus.

There are, however, reasons for doubting that the patterns of sounds reaching the two tympana are the only critical factors, at least in what concerns lateral asymmetry. Morais and Bertelson (1975) found lateral asymmetry in a stereophonic listening situation in which there was no systematic intensity difference between the two messages in each ear but only a time delay in order to produce an impression of lateralization. On the other hand, there are data showing that gaze direction can affect lateral asymmetry in a dichotic monitoring task: Gopher (1971, cited by Kahneman, 1973) has shown that performance on messages delivered to one ear can be improved relative to the other ear by shifting the gaze in the corresponding direction. Lastly, one of us (Morais, 1974-1975), working with a situation where the listener was exposed to two simultaneous messages from two hidden loudspeakers, one on his left and one on his right, has found that the better identification of the message coming from the right could be reduced by suggesting, through the use of a dummy loudspeaker, that the separation between the sources was smaller than it actually was. Patterns of sounds at the ears are thus not the sole determinant of lateral asymmetry.

The determinants of frontal-position advantage are, however, still unknown, and a purely acoustical interpretation is an open possibility. In all studies in which the effect has been observed, the subjects sat on a stool with head, eyes, trunk, and limbs all oriented in the same direction. This posture will be called here "the homogeneous posture." It is probably the most usual posture in real-life situations when one is listening to speech. Now, it is clear that if frontal position advantage is determined exclusively by acoustical factors, the head should be the only segment of the body whose orientation should affect the effect. If, on the other hand, and as seems to be the case for lateral asymmetry, there is some place for determinants at more central levels, one might expect that orientation of other segments, such as gaze or trunk, might play some role as well.

In the following experiments, the subjects were exposed to two messages from two spatially separated loudspeakers and asked to report one of them. The methodology consisted of having the subject adopt, in some conditions, the homogeneous posture, with one loudspeaker in front and the other in a less favorable location, and in other conditions, nonhomogeneous postures in which one or more segments were shifted laterally towards the second loudspeaker. Experiment 1 explored the effect of turning head and eyes laterally. Experiment 2 examined separately the effects of turning the eyes, the head, and the trunk and limbs.

\section{EXPERIMENT 1}

This experiment was designed to examine the influence of head and eye orientation on two spatial effects demonstrated in earlier studies: the advantage of a frontal source over one at $90 \mathrm{deg}$ to the left and the other at $90 \mathrm{deg}$ to the right (Hublet et al., 1976; Morais \& Bertelson, 1973). For each comparison, two conditions were run. In one, the subject adopted the homogeneous posture, and was thus oriented towards one loudspeaker with the other at $90 \mathrm{deg}$ either to his left or to his right. In the other condition, the subject turned his head and eyes toward the lateral loudspeaker, keeping his trunk and his limbs facing the frontal loudspeaker.

If the head orientation was the sole factor responsible for the spatial effects that have been described above, turning the head to the left by about $90 \mathrm{deg}$ should create an advantage for the loudspeaker situated on the left (with regard to the subject's trunk) as great as that of the frontal loudspeaker over the right one, with the homogeneous posture. A corresponding prediction exists for the two other conditions in the experiment.

\section{Method}

Material and experimental situation. The tape employed in this experiment was provided by C. Darwin and was prepared with a parallel formant speech synthesis program on the Elliott 4130 computer at the Department of Experimental Psychology of the University of Sussex. It contained 300 pairs of simultaneous consonant-vowel (CV) syllables selected from the set /ba, da, ga, pa, ta, ka/. Each syllable lasted $300 \mathrm{msec}$. One pair occurred every $5 \mathrm{sec}$. The two syllables of each pair were recorded on different tracks, at a pitch of $100 \mathrm{~Hz}$ on one track and at $161 \mathrm{~Hz}$ on the other; they were never tokens of the same syllable. Each of the 15 different pairs of syllables occurred 20 times under each of the two possible distributions between the tracks. A short tape, on which each of the 6 syllables was recorded four times in random order, simultaneously on both tracks, 1 syllable every $5 \mathrm{sec}$, also was prepared for use in a preliminary screening test.

The material was played on a Revox A 77 tape recorder at an intensity level of about $70 \mathrm{~dB}$ (SPL). Each channel of the tape recorder was connected to one of two loudspeakers (Isophon HSB 15/8). Three loudspeakers, positioned at head level, occupied positions in front of the subject $(0 \mathrm{deg}), 90 \mathrm{deg}$ to the left, and $90 \mathrm{deg}$ to the right, at $1 \mathrm{~m}$ from the nearest ear. Two of them were used in each condition. Sound reflections from the silent loudspeaker did not produce any significant change in intensity, compared with a situation in which the loudspeaker was removed, as measured by a measuring amplifier (Brüel and Kjaer 2606) from a condenser microphone cartridge (Brüel and Kjaer 4135) placed at ear level.

The room was quiet, though not soundproof. The subject sat on a stool with a fixed backrest and wore a headlight with a 
narrow beam which provided a control for the orientation of his head. The stool occupied the same position in the room for all subjects, since previous experiments run in the same room (Hublet, Morais, \& Bertelson, 1976, 1977) had shown that sitting with the sagittal plane along the longest horizontal dimension or along the shortest one had no differential effect on performance or on spatial differences.

Procedure. There were four conditions, depending on the location of the two loudspeakers that delivered the stimuli and on the orientation of the head. In two conditions, the subject sat in the homogeneous posture; in one of them, the messages came from the frontal and the left loudspeakers (condition L); in the other condition, they came from the frontal and the right loudspeakers (condition $\mathrm{R}$ ). In the third condition (condition H-right), the messages came from the frontal and the right loudspeakers, but the head of the subject faced the loudspeaker on the right. In a fourth condition (condition $\mathbf{H}$-left), the messages came from the frontal and the left loudspeakers, but the head of the subject faced the loudspeaker on the left. In the last two conditions, gaze went with head, but shoulders, trunk, and limbs remained oriented towards the frontal loudspeaker.

The subjects were instructed to keep the beams of their headlights, during presentations, on a small colored circle $(.8 \mathrm{~cm}$ diam) that was posted on the frontal loudspeaker for conditions $L$ and $R$, on the loudspeaker on the right for condition H-right, and on the loudspeaker on the left for condition $\mathrm{H}$-left.

The session consisted of 44 practice trials and 256 experimental trials, preceded by a screening test. For the latter, the subject adopted the homogeneous posture and had to identify 24 syllables delivered simultaneously through the two lateral loudspeakers. The subjects who made more than five errors in this test were eliminated. The practice trials were presented under the condition which was to be run first. They consisted of two groups of 22 trials defined by the position of the source the subject should listen to. The experimental trials were grouped in four blocks of 64 trials, one block under each of the four conditions. Each block was divided into four runs of 16 trials. The subject was told, before each run of trials, the position of the source he should listen to, and was instructed to report only the syllable from that source. He was told to write down the syllable on a response sheet immediately after the trial; he was encouraged to guess when not sure. After the first practice group of trials and after each group of trials within an experimental block, the report instructions were shifted and, at the same time, the loudspeakers were reversed; consequently, the subjects were listening to the same loudspeaker and to the same track of the tape (the one with the $161-\mathrm{Hz}$ pitch) across the whole experiment.

Each of four different orders of presentations of the four conditions, determined by a Latin square balanced for sequential effects, was assigned to four subjects. Two of the four subjects in the same line listened to the source in one or the other of the two positions in each condition in the order 1212; for the other two subjects, the order was 2121 . One of the two subjects with the same order of presentation of conditions and same order of listen- ing instructions received the two syllables of each trial at the same intensity, and the other subject received the syllable to be reported at an intensity $6 \mathrm{~dB}$ higher than the other syllable.

Subjects. Sixteen right-handed students who reported no hearing defect were tested. Six were male and 10 female. Their ages ranged from 17 to 31 years. Each took part in one 60-min session.

\section{Results and Discussion}

The mean percentages of correct responses in the four conditions and for each source, and the distribution of subjects according to the source for which they had more correct responses in each condition, are shown in Table 1.

Performances for the two sources in each condition were compared by means of a score of nonhomogeneity, the $f$ score, proposed by Marshall, Caplan, and Holmes (1975). This score was computed according to the formulas $\left.\left[\mathrm{F}_{\mathrm{c}}-\mathrm{L} \text { (or } \mathrm{R}\right)_{\mathrm{c}}\right] /$ $\left.\left[F_{c}+L \text { (or } R\right)_{c}\right]$, when percentage of correct responses averaged over the two sources was smaller than $50 \%$, and $\left.\left.\left[F_{c}-L \text { (or } R\right)_{c}\right] /\left[F_{E}+L \text { (or } R\right)_{E}\right]$, when it was greater (where $F$ is the frontal loudspeaker with regard to the trunk, $\mathrm{L}$ the left one, $\mathrm{R}$ the right one, $\mathrm{C}$ the correct responses, and $\mathrm{E}$ the errors). Although relative intensity of the two simultaneous messages affected overall performance, it does not seem to have had great influence on the spatial position effect. So that variable is not considered in Figure 1, in which the mean $f$ scores for the four conditions are shown. When the subject turned his head to the right, there was only a small nonsignificant advantage of the $\mathbf{R}$ source, contrasting with the clear advantage of the F source over the L source when he adopted the homogeneous posture. The advantage observed for the F source over the $R$ source with the subject in the homogeneous posture disappeared but was not reversed when he turned his head to the left. According to the hypothesis of an exclusive role of head orientation, both the sum of $f$ scores for conditions $\mathrm{L}$ and $\mathrm{H}$-right and the sum of $\mathrm{f}$ scores for conditions $\mathrm{R}$ and $\mathrm{H}$-left should not be significantly different from zero. We estimated, for each subject, the average of these two sums and found that 13 subjects had positive values and 3 had negative ones. This pattern is significant by a two-tailed sign test at $p=.022$. Orientation of the

Table 1

Experiment 1: Mean Percentages of Correct Responses by Condition and by Source, and Distribution of Subjects According to the Source for Which They Had More Correct Responses in Each Condition

\begin{tabular}{|c|c|c|c|c|c|c|c|c|}
\hline \multirow[b]{3}{*}{ Source } & \multicolumn{8}{|c|}{ Condition } \\
\hline & \multicolumn{2}{|c|}{ L } & \multicolumn{2}{|c|}{ H Right } & \multicolumn{2}{|c|}{$\mathbf{R}$} & \multicolumn{2}{|c|}{ H Left } \\
\hline & $\mathrm{F}$ & L & $\mathbf{F}$ & $\mathbf{R}$ & $\mathbf{F}$ & $\mathbf{R}$ & $\mathbf{F}$ & $\mathbf{R}$ \\
\hline $\begin{array}{l}\text { Group Same Intensity } \\
\text { Group } 6 \mathrm{~dB} \text { Louder } \\
\text { Total Sample }\end{array}$ & $\begin{array}{l}42.1 \\
59.4 \\
50.8\end{array}$ & $\begin{array}{l}34.8 \\
46.9 \\
40.9\end{array}$ & $\begin{array}{l}35.2 \\
49.6 \\
42.4\end{array}$ & $\begin{array}{l}41.4 \\
52.8 \\
47.1\end{array}$ & $\begin{array}{l}42.6 \\
54.3 \\
48.5\end{array}$ & $\begin{array}{l}37.1 \\
48.5 \\
42.8\end{array}$ & $\begin{array}{l}39.1 \\
50.4 \\
44.8\end{array}$ & $\begin{array}{l}37.1 \\
51.6 \\
44.4\end{array}$ \\
\hline $\mathrm{N}^{*}$ & 14 & 2 & 5 & 10 & 11 & 5 & 7 & 7 \\
\hline
\end{tabular}

*Number of subjects with more correct responses for the corresponding source. 
Condition L

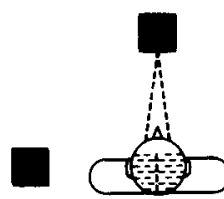

$$
\begin{gathered}
f=12.3 \\
t=4.06 \\
d f=15 \\
P<0.005
\end{gathered}
$$

Condition $\mathrm{H}$ right

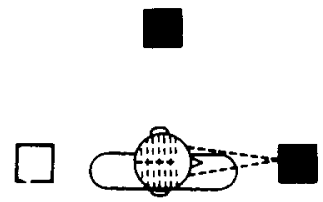

$$
r=-3.6
$$$$
t=-0.78
$$$$
d f=15
$$

NS
Condition $\underline{R}$

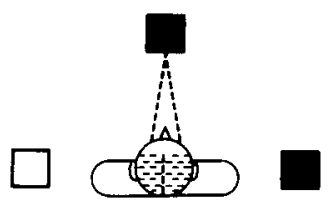

$$
\begin{gathered}
r=6.9 \\
t=1.90 \\
d r=15 \\
P<0.05
\end{gathered}
$$

Condition $\mathrm{H}$ left

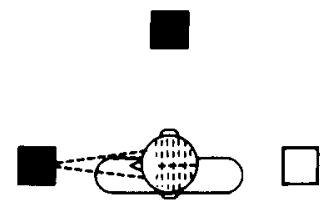

$$
\begin{aligned}
r & =-0.6 \\
t & =-0.12 \\
d f & =15
\end{aligned}
$$

NS

Figure 1. Experiment 1 : Mean $f$ scores for each condition and the corresponding $t$ tests.

head is thus not the only factor determining the advantage of a frontal source over a lateral one. Orientation of trunk and limbs seems to play an effective role as well.

\section{EXPERIMENT 2}

A more detailed analysis of the role of posture in frontal position advantage was undertaken in Experiment 2 . Three loudspeakers, separated by $35 \mathrm{deg}$ in azimuth, were in view. Whatever the condition, two messages, one from the left and one from the middle loudspeaker, were presented on each trial and the subject was instructed beforehand to report one of them. In the control condition, the subject adopted the homogeneous posture, oriented toward the middle loudspeaker. In three other conditions, the gaze, the head, and the trunk and limbs were independently shifted towards the left loudspeaker, the other segments remaining oriented towards the middle one. The advantage of the middle loudspeaker was expected to be reduced in each of these conditions relative to the control condition. Experiment 1 has shown that direction of head and gaze is an important determinant of directional effects. The relative contribution of head and of gaze is not known. Gopher's results, mentioned in the introduction, show that gaze direction can be a nonnegligible factor. On the other hand, trunk-and-limb orientation is probably not negligible either, since, in Experiment 1, the spatial effect did not reverse when head and gaze were shifted to the left or to the right.

Evidence was sought also about the way the orientation of the different segments combine their effects. For that purpose, two additional conditions were included in which (1) head and gaze, and (2) head, gaze, and trunk and limbs all turned to the left.

A task was chosen which, for the subject in the homogeneous posture, combined inputs from a loud- speaker in front of the subject and one to his left, because it is the arrangement which, for a given angular separation, produces the strongest frontal position advantage. The angle of $35 \mathrm{deg}$ was chosen because it is about the larger one by which the gaze can be deviated from the frontal direction for some time without causing discomfort. The third silent loudspeaker to the right was included to make the situation as symmetrical as possible.

The mode of responding was also manipulated in this experiment. In the previous studies, the subjects had to write their responses on a sheet, and one might wonder whether an activity performed by the upper limb of one side could not influence auditory spatial effects. Here, half the subjects wrote their responses and half gave them orally.

\section{Method}

Material and experimental situation. The same tape used in Experiment 1 was employed here. Each channel of the tape recorder was connected to one of two loudspeakers which were positioned, at head level, one in front of the subject $(0 \mathrm{deg})$ and the other $35 \mathrm{deg}$ to the left at $1 \mathrm{~m}$ distance from the nearest ear. A third loudspeaker, unconnected, was put at $35 \mathrm{deg}$ to the right and at the same distance from the subject. As in Experiment 1, the subject sat on a stool with a fixed backrest and he wore a headlight providing a control for the orientation of his head. A video camera (Sony AVC-3250 CES/CEK), positioned below the middle loudspeaker, was connected to a screen on which the experimenter could monitor the eyes of the subject. The experimenter sat at a table which was situated behind the subject at a distance of $1.5 \mathrm{~m}$.

Procedure. There were six conditions, depending on head posture, gaze direction, and trunk-plus-limbs posture. In all of them, two simultaneous messages occurred on each trial, one coming from the middle loudspeaker $(\mathrm{M})$ and the other from the loudspeaker on the left (L). The message the subject was instructed to listen to was delivered at an intensity $6 \mathrm{~dB}$ higher than the other message. There was a control condition (condition $\mathrm{C}$ ), in which the subject adopted the homogeneous posture, and five experimental conditions. In condition $H$, the head of the subject faced the $L$ loudspeaker, but his eyes, trunk, and limbs remained oriented towards the $M$ loudspeaker. In condition $G$, the subject looked at the L loudspeaker, but his head, trunk, and limbs 
remained oriented towards the $M$ loudspeaker. In condition $T$, the trunk and limbs of the subject, as well as the stool on which he sat, were oriented towards the L loudspeaker, but his head and eyes remained oriented towards the $M$ loudspeaker. In condition HG, the head and eyes of the subject were oriented towards the L loudspeaker, but his trunk and limbs remained oriented towards the M loudspeaker. Finally, in condition HGT, the head, eyes, trunk, and limbs of the subject, as well as the stool on which he sat, were all oriented towards the L loudspeaker.

The subjects were instructed to look at a small colored circle $(.8 \mathrm{~cm}$ diam) that was posted just below the $M$ loudspeaker for conditions $\mathrm{C}, \mathrm{H}$, and $\mathrm{T}$ and just below the $\mathrm{L}$ loudspeaker for conditions G, HG, and HGT. Gaze direction was controlled through the monitor, and for this purpose the experimenter adjusted the position of the camera before each condition. The headlight was put on the head of the subject in such a way that its beam was kept on the middle of the superior edge of the loudspeaker his head was facing.

Each of the two sessions consisted of 36 practice trials and 264 experimental trials. At the beginning of the first session, the subject was given a screening test, in which he had to identify 24 successive syllables, each delivered simultaneously through the $\mathbf{M}$ and the $\mathrm{L}$ loudspeakers. The subjects who made more than five errors on this test were eliminated. Sixteen practice trials were presented under the condition to be run first, and four practice trials were presented under each of the other conditions. Each of these groups of practice trials immediately preceded the corresponding condition. The experimental trials were grouped in six blocks of $\mathbf{4 4}$ trials corresponding to the six conditions, and within each block were grouped in four runs of 11 trials each. Before each run of trials, the experimenter pointed to the loudspeaker the subject was to listen to. The subject was instructed to report only the syllable from that source. Half the subjects wrote down the syllable on a response sheet immediately after the trial; the other half pronounced it aloud, also immediately after the trial; in both cases, they were encouraged to guess when not sure. After the first half of each group of practice trials, and after each run of trials within an experimental block, the instructions about the position to report were shifted and, at the same time, the $\mathrm{M}$ and $\mathrm{L}$ loudspeakers were reversed; as a consequence, the subjects were listening to the same loudspeaker and to the same track of the tape (track 2, where the pitch was $161 \mathrm{~Hz}$ ) across the whole experiment.

Six different orders of presentation of the six conditions were determined by a Latin square balanced for sequential effects. Two subjects of each group of 12 with the same response mode were assigned to each of those orders of presentation, one listening to the two positions in each condition in one order (for instance, MLML), the other in the opposite order. The order of attention instructions was reversed in the second session for each subject.

Subjects. Twenty-four right-handed students who reported no hearing defect were tested. Nine were male and 15 female. Their ages were in the range of $19-32$ years. Each took part in two 60 -min sessions held on different days. Six of the subjects had participated in Experiment 1 and had shown frontal position advantage.

\section{Results}

Table 2 shows the mean percentages of correct responses for each source in the six conditions. It can be seen that performance averaged over the two sources did not vary largely as a function of condition: it ranged from $53.7 \%$ in condition $C$ to $57 \%$ in condition T. ${ }^{1}$

The distribution of subjects according to the source for which they had more correct responses in each condition is shown in Table 3; the superiority of the
Table 2

Experiment 2: Mean Percentages of Correct Responses by Condition and by Source

\begin{tabular}{lccc}
\hline & L & M & Mean \\
\hline C & 47.7 & 59.7 & 53.7 \\
H & 53.2 & 55.9 & 54.6 \\
G & 53.9 & 57.2 & 55.6 \\
T & 55.0 & 59.0 & 57.0 \\
HG & 54.7 & 57.8 & 56.3 \\
HGT & 54.7 & 55.9 & 55.3 \\
\hline
\end{tabular}

Table 3

Experiment 2: Distribution of Subjects According to the Source for Which They Had More Correct Responses in Each Condition

\begin{tabular}{lrrrrrr}
\hline Source & C* $^{*}$ & H & G & T $\dagger$ & HG & HGT \\
\hline M & 20 & 15 & 12 & 18 & 14 & 12 \\
L & 2 & 8 & 8 & 4 & 8 & 10 \\
None & 2 & 1 & 4 & 2 & 2 & 2 \\
\hline
\end{tabular}

${ }^{*} p<.001 . \quad t_{p}<.002$.

$M$ source over the $L$ one reached statistical significance, by unilateral sign tests, in conditions $\mathrm{C}$ and $\mathrm{T}$, but not in the other conditions.

Computation of $f$ scores on correct responses was done here using the same formulas as in Experiment 1 but with the $M$ loudspeaker in place of the $F$ one. The mean $f$ scores for subjects who wrote their responses, for subjects who gave them orally, and for the total sample, in each condition, are shown in Figure 2. Frontal position advantage was clearly present in condition $C$. In the other conditions, the advantage of the middle source was much smaller. Differences between these conditions were rather slight. A threeway analysis of variance, including conditions, mode of responding, and subjects (hierarchic model), was performed on $f$ scores. Condition was significant at $p<.01[F(5,22)=3.42]$. Mode of responding and the interaction of Condition by Mode of responding were both nonsignificant $[F(1,22)=3.49$ and $F(5,110)$ $=1.12$, respectively].

Five independent planned comparisons were performed. Three of them addressed the question of whether or not each of the segments that have been considered here contributed to the frontal position advantage that was observed with the subject oriented entirely towards the middle loudspeaker. Comparisons $H$ vs. $C, G$ vs. $C$, and $T$ vs. $C$ were significant at $\mathrm{p}<.001[\mathrm{t}(110)=3.37], \mathrm{p}<.005[\mathrm{t}(110)=2.71]$, and $\mathrm{p}<.025[\mathrm{t}(110)=2.27]$, respectively. These results imply that each of the factors-gaze direction, head posture, and trunk-and-limb posture-does contribute to frontal position advantage. Two other comparisons addressed the question of whether or not the effect of one particular segment combined in an additive way with the effect of another segment. Comparison HG-H vs. G-C was significant at 


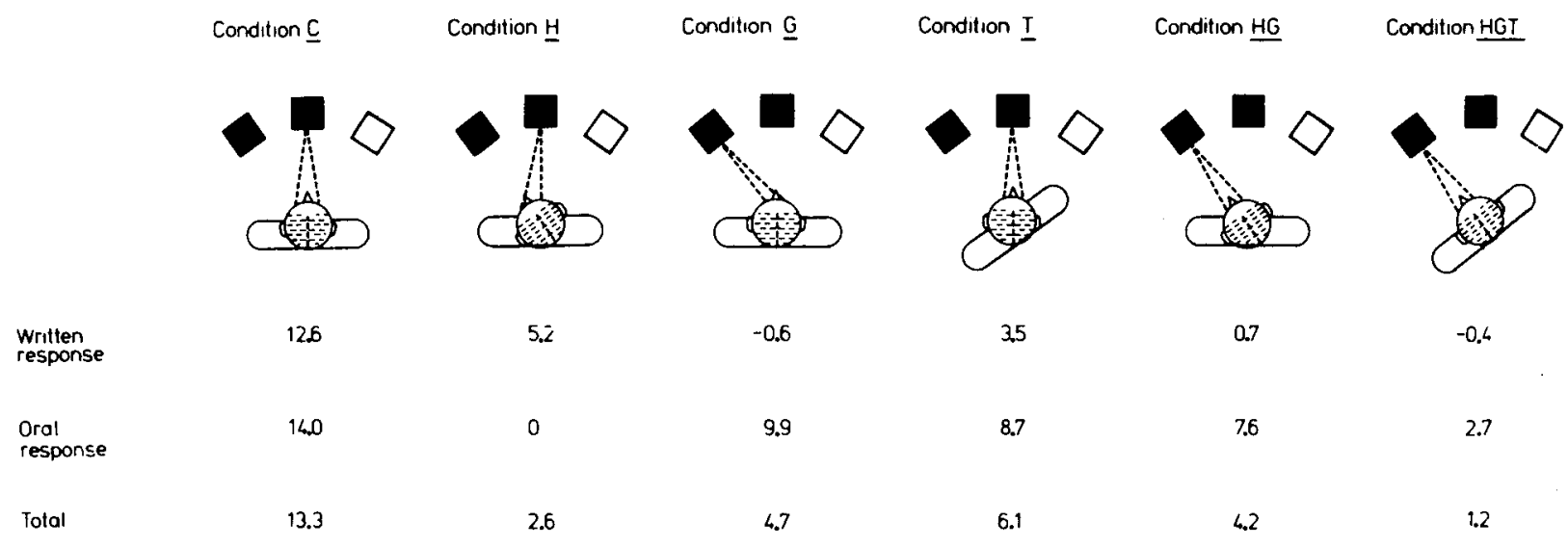

Figure 2. Experiment 2: Mean f scores for each condition, by mode of responding and for the total sample.

$\mathrm{p}<.05$ by a two-tailed test $[\mathrm{t}(110)=2.276]$, but comparison HGT-HG vs. T-C was nonsignificant $[\mathrm{t}=.937]$. Thus, while gaze orientation did not combine in an additive way with head orientation, the hypothesis of additivity could not be rejected for segments below and above the neck. However, if we had asked whether turning all the body adds something to the effect of turning only head and gaze (comparison HGT vs. HG), instead of testing full additivity, we would not have rejected the null hypothesis either $[t=.94]$.

\section{GENERAL DISCUSSION}

For a listener sitting in what has here been called the homogeneous position, that is, with gaze, head, and body all oriented in the same direction, attention can be more effectively focused on a source situated to his front than on one to one side. This frontal position advantage, first observed by Morais and Bertelson (1973), was replicated in the two experiments by Hublet et al. (1976, 1977), in the present two experiments, and in an as yet unpublished study (Morais, Hublet, \& Bertelson, Note 1), and is thus a consistent phenomenon.

The present study was planned mainly to separate the contributions of gaze, head, and body (trunk plus limbs) orientations to the effect. Experiment 1 showed that, in addition to the important influence of head and gaze orientation, body orientation plays a detectable role. Experiment 2 showed that each of the three segments is important: when gaze alone, head alone, or body alone was turned towards a lateral source, focusing on that source was made more effective and focusing on the frontal source less effective.

Secondarily, it was attempted, in Experiment 2, to obtain information regarding the rules governing the combination of the different effects. According to an additive model, each segment would produce its own pattern of directional effect, and a global perfor- mance difference would result from a linear combination of these components. Under such a hypothesis, the reduction in frontal position advantage observed with one segment shifted to the left would be due to the fact that the contribution of that segment to performance difference changed from frontover-left advantage in favor of the middle loudspeaker to front-over-right advantage in favor of the left loudspeaker. As far as only the segments above the neck are concerned, Experiment 2 provided clear evidence against the additive model: the effect of shifting gaze when the head was already turned to the side was significantly inferior to the effect of shifting gaze alone. On the other hand, additivity between body and head-and-gaze effects could not be rejected. It must be noted, however, that shifting the body also (condition HGT) did not produce any significant increment relative to the head-gaze effect. These inconclusive statistical results might be related to the smallness of the reduction in the advantage of the middle loudspeaker when only the body was shifted. In fact, the latter condition was the only one, besides the control condition, to show a significant advantage of the middle loudspeaker.

The fact that the orientation of the head is not the only factor in determining frontal position advantage eliminates any interpretation of the effect based exclusively on physical differences at the ear between the signals from the two loudspeakers. The phenomenon is, partly at least, caused at a more central level where auditory data interact with data of visual and proprioceptive origin relevant to the subject's orientation. No acoustical effect external to the listener would account for the significant reduction in the advantage of the middle loudspeaker that was observed when gaze alone or trunk alone were shifted to the side.

Regarding the nature of the determinants at more central levels considered in the preceding paragraph, it is tempting to relate them to the postures people 
spontaneously adopt when gathering visual and auditory information. The effect of gaze direction, for instance, might have derived from the fact that we normally look at the source of sounds we are paying attention to. In many cases, as in speech perception, for instance, we thus obtain, through sight, information which helps in interpreting the auditory data. Even when no useful visual data are available, the tendency to look in the direction of the source of sounds is manifest. When our subjects were told, before a block of trials, to report the syllable delivered on the lateral loudspeaker while looking at the frontal one, they sometimes glanced at the first before fixating the second. In a similar vein, Gopher (1973) has shown that subjects exposed to dichotic stimulation and instructed to focus attention on one ear tend to make a large saccade towards that ear at the beginning of the message. In an unpublished experiment by one of us (Morais), in which subjects were exposed to simultaneous messages from one loudspeaker in front of them and from one behind them, it was observed that they looked firmly at the frontal loudspeaker when they were instructed to listen to it, but tried to avoid it and looked to the side, downwards, upwards, or even closed their eyes when they were asked to listen to the source in the rear. There is, thus, ample evidence that looking tends to accompany auditory attention, even when no useful visual data are obtainable. The results of Gopher (1971, cited by Kahneman, 1973), referred to in the introduction, and those of the present study show that looking actually facilitates the focusing of auditory attention. Several of our subjects spontaneously commented that they found it difficult to pay attention to a loudspeaker they were not allowed to look at.

A similar coordination with attention focusing may be present in the case of both head and body orientation. When attending to an auditory source, we not only tend to look in its direction, but we often also orient the head and the body in such a way as to bring the source into a frontal direction.

Data have recently become available showing that a tendency to orient the head in relation to the source of a sound can be detected even in newborn babies (Alegria \& Noirot, 1978). As has already been pointed out, the advantage gained through head orientation might be of purely acoustical origin and be due to unimpaired access to both ears for sounds from a source straight ahead. It might, however, also have to do with auditory-visual coordination: The gaze, with its accompanying facilitating effect on attention, can most comfortably be centered on a straightahead target and, also, can then most easily follow the target if it is moved in either direction. Whatever the original, or primary, advantage gained from head orientation, long-time practice in putting auditory targets straight ahead may have produced a linkage between head orientation and attention focusing.
The case of body orientation is, in a way, simpler, since the only primary advantage which can be gained from orienting the body towards a source is comfort and facility in orienting the superior segments, head and gaze. In the situation of Experiment 2, however, with an angle between the sources well within the range of comfortable head or gaze shifts, this factor could not produce an effect. The most plausible explanation of the observed effect is then an influence of body orientation on attention focusing, derived from long-time practice in orienting the body.

One important question can be asked regarding the conditions of occurrence of those effects of orienting the different segments: Do they depend on the fact that the orientation is obtained through voluntary adjustments? Would, for example, the effects of orienting the head or the body be obtained if the subject was restrained in a chair and his body and his head put in the desired position without instructions? The possibility exists that it is the voluntary activity involved in keeping one or several segments oriented toward the lateral source which biases attention toward that source. Whether it is posture per se or posture keeping activity which is critical in producing the effects cannot be decided on the basis of the present results. Yet, the finding that the effects of shifting the different segments do not add in a linear fashion could be taken as favoring the second alternative: If the factor which biases attention toward a particular location is simply the fact of having to do some postural orientation in its direction, we can expect the number of segments which must be oriented not to matter too much.

One surprising aspect of the results has not been considered so far-the fact that in condition HGT the left loudspeaker towards which the subject was oriented in the homogeneous position did not give the best performance. In view of earlier results (Hublet et al., 1976) showing an advantage, albeit small, for a frontal source over one $45 \mathrm{deg}$ to the right, an advantage for that loudspeaker would have been expected in that condition. If the finding is confirmed in new studies, it might mean that, besides actual egocentric relations, representation by the subject of the spatial relation between sources and frame of reference plays some role in determining attention biases.

\section{REFERENCE NOTE}

1. Morais, J., Hublet, C., \& Bertelson, P. Constraints on auditory attention focusing: Environmental sounds versus speech. Manuscript in preparation.

\section{REFERENCES}

Alegria, J., \& Noirot, E. Neonate orientation behaviour towards human voice. International Journal of Behavioral Development, 1978, 1, 291-312.

Curry, F. K. W. A comparison of left-handed and right-handed subjects on verbal and non-verbal dichotic listening tasks. Cortex, 1967, 3, 343-352. 
GOPHER, D. Eye-movement patterns in selective listening tasks of focused attention. Perception \& Psychophysics, 1973, 14, 259-264.

Hublet, C., Morais, J., \& Bertelson, P. Spatial constraints on focused attention: Beyond the right-side advantage. Perception, $1976,5,3-8$.

Hublet, C., Morais, J., \& Bertelson, P. Spatial effects in speech perception in the absence of spatial competition. Perception, 1977, 6, 461-466.

Kahneman, D. Attention and effort. Englewood Cliffs, N.J: Prentice-Hall, 1973.

Kimura, D. Cerebral dominance and the perception of verbal stimuli. Canadian Journal of Psychology, 1961, 15, 166-171.

Kimura, D. Left-right differences in the perception of melodies. Quarterly Journal of Experimental Psychology, 1964, 16, 355-358.

Kimura, D. Functional asymmetry of the brain in dichotic listening. Cortex, 1967, 3, 163-178.

Marshall, J., Caplan, D., \& Holmes, J. The measure of laterality. Neuropsychologia, 1975, 13, 315-321.

Morais, J. The effects of ventriloquism on the right-side advantage for verbal material. Cognition, 1974-1975, 3, 127-139.

Morais, J., \& Bertelson, P. Laterality effects in diotic listening. Perception, 1973, 2, 107-111.
Morais, J., \& Bertelson, P. Spatial position versus ear of entry as determinant of the auditory laterality effects: A stereophonic test. Journal of Experimental Psychology: Human Perception and Performance, 1975, 104, 253-262.

Sparks, R. W., \& Geschwind, N. Dichotic listening in man after section of neocortical commissures. Cortex, 1968, 4, 3-16.

Studdert-Kennedy, M., \& Shankweiler, D. Hemispheric specialization for speech perception. Journal of the Acoustical Society of America, 1970, 48, 579-594.

\section{NOTE}

1. One referee questioned whether or not some differences could have arisen between conditions as a consequence of different sound reflections when the shoulders were in the axis of the ears (conditions $\mathrm{C}, \mathrm{G}$, and HGT) and when they were not (conditions $\mathrm{H}, \mathrm{T}$, and $\mathrm{HG}$ ). That was not the case, at least concerning overall performance.

(Received for publication June 26, 1979; revision accepted December 26, 1979.) 